\title{
Prevalencia de las enfermedades alérgicas y su asociación con la lactancia materna y el inicio de la alimentación complementaria en niños de edad escolar de Ciudad Guzmán, México \\ Prevalence of allergic diseases and their association with \\ breastfeeding and initiation of complementary feeding in \\ school-age children of Ciudad Guzmán, Mexico
}

\author{
Dr. Jaime Morales-Romero ${ }^{a}$, Dr. Martín Bedolla-Barajas ${ }^{b}$, Dra. Laura López-Vargas ${ }^{c}$ \\ y Dr. Enrique Romero-Velarded
}

\section{RESUMEN}

Introducción. El efecto que tiene la lactancia materna y la alimentación complementaria sobre la prevalencia de las enfermedades alérgicas ha mostrado resultados inconsistentes.

Objetivo. Evaluar el efecto dela lactancia materna y el momento de inicio de la alimentación complementaria en la prevalencia de las enfermedades alérgicas.

Población y métodos. Estudio transversal analítico con base poblacional realizado en niños de 6 a 12 años de edad inscriptos en escuelas primarias, seleccionados mediante muestreo polietápico. Se aplicó a sus padres o tutores un cuestionario estructurado para identificar enfermedades alérgicas (asma, rinitis alérgica o dermatitis atópica), antecedente de lactancia materna prolongada y edad de inicio de la alimentación complementaria. La búsqueda de asociaciones entre variables se realizó mediante regresión logística.

Resultados. Se incluyeron 740 niños. La frecuencia de lactancia $\geq 6$ meses fue $73,4 \%$ y de alimentación complementaria con $\leq 4$ meses fue $31,9 \%$. La lactancia materna prolongada no tuvo efecto sobre la prevalencia de las enfermedades alérgicas. Se observó un efecto protector en la frecuencia de la dermatitis atópica cuando el inicio de la alimentación complementaria era tardío, OR ajustado=0,36; IC95\%:0,1-0,8 (p 0,019). Conclusiones. El efecto protector de la lactancia materna sobrela prevalencia delas enfermedades alérgicasnopudosercomprobado.Laprevalencia de dermatitis atópica es menor con un comienzo tardío de la alimentación complementaria.

Palabras clave: lactancia materna, niño, asma, rinitis alérgica, dermatitis atópica.

http:/ /dx.doi.org/10.5546/aap.2015.324

\section{INTRODUCCIÓN}

Las enfermedades atópicas son problemas de salud importantes durante la niñez, que han incrementado su prevalencia en amplias regiones del mundo. ${ }^{1-4}$ Los factores de riesgo que han tratado de explicar este comportamiento son múltiples, entre ellos están la influencia de la lactancia materna y el momento de inicio de la alimentación complementaria. Evaluar el efecto protector de la lactancia materna en el desarrollo de las enfermedades alérgicas no es tema reciente. Grulee y Sanford, ${ }^{5}$ en 1936, documentaron que la lactancia materna era un factor protector de la dermatitis atópica, y desde entonces, diversas investigaciones subsecuentes han mostrado resultados contradictorios. Existe evidencia de que la lactancia materna disminuye la frecuencia de las enfermedades alérgicas, ${ }^{6}$ pero también se ha observado que dicho tipo de alimentación facilita su presentación, 7 que no contribuye en la prevención de la atopia ${ }^{8}$ o tiene beneficio transitorio. ${ }^{9}$

Respecto a la alimentación complementaria y su asociación con las enfermedades alérgicas, los resultados vertidos señalan que la introducción tardía a la dieta de los lactantes no previene el desarrollo de las enfermedades alérgicas ${ }^{10,11} \mathrm{O}$ la sensibilización a alérgenos. ${ }^{12}$ Una revisión sistemática de este tema arrojó resultados inconsistentes. ${ }^{13}$

De acuerdo con nuestro conocimiento, en México, no hay información publicada que demuestre el beneficio de la lactancia materna o el retraso de la introducción de alimentos a la dieta de los niños y 
la prevención de las enfermedades alérgicas. Este estudio forma parte de una investigación epidemiológica más amplia realizada en una ciudad localizada en el occidente de México.

Nuestro objetivo fue evaluar el efecto de la lactancia materna y el momento de inicio de la alimentación complementaria en la prevalencia de las enfermedades alérgicas en una muestra de niños en edad escolar.

\section{POBLACIÓN Y MÉTODOS}

Estudio de corte transversal y analítico; los datos fueron recolectados de abril a julio de 2008.

\section{Escenario}

Ciudad Guzmán es una población urbana localizada al sur del estado de Jalisco, México. La actividad económica de la región está constituida por la oferta de servicios, la industria y las actividades agropecuarias.
La metodología de nuestro estudio ya fue previamente descrita en otro trabajo publicado. ${ }^{14}$ Con base poblacional, se incluyeron niños y niñas de 6 a 12 años de edad, inscritos en escuelas de educación primaria, del turno matutino y vespertino, así como del orden público y privado.

Como base para el cálculo del tamaño de la muestra, partimos de la prevalencia de asma esperada en niños escolares (7\%), un nivel de confianza del $95 \%$ y una precisión del $2 \%$. El tamaño muestral fue de 626 niños; después de ajustar a $20 \%$ de posibles pérdidas, el tamaño final fue de 752 .

\section{Selección de la muestra}

Para conformar el grupo de estudio, se utilizó un muestreo probabilístico, estratificado y por conglomerados en tres etapas (Figura 1).

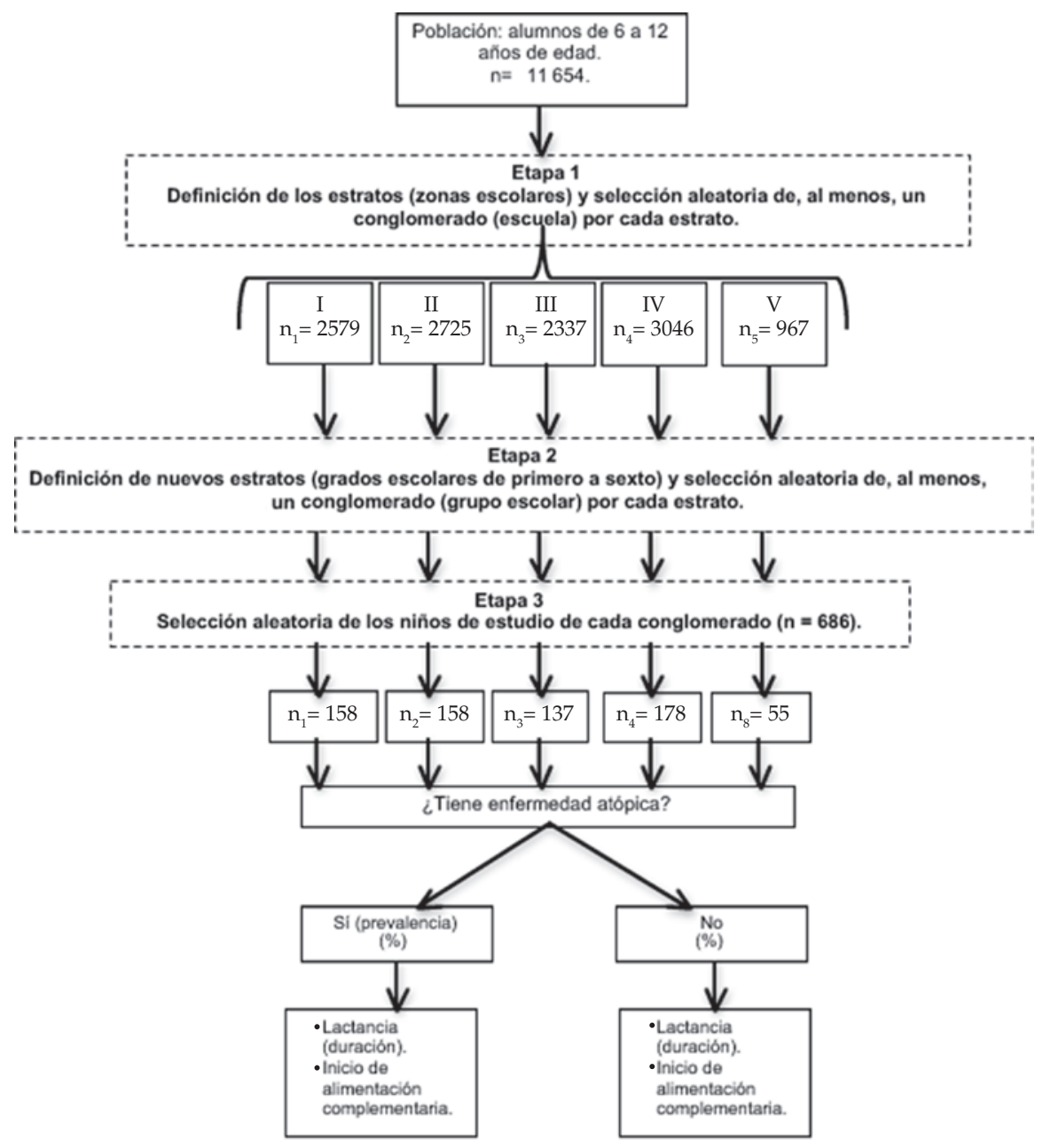




\section{Etapa 1}

Las zonas escolares fueron consideradas estratos y, en cada uno, se calculó una submuestra. Para decidir el número de escuelas (conglomerados) por seleccionar de cada estrato, se multiplicó el porcentaje de alumnos con el que contribuyó cada zona escolar por el número de escuelas de cada zona, respectivamente. Las escuelas fueron seleccionadas de manera probabilística.

\section{Etapa 2}

En este nivel, cada uno de los grados escolares (de primero a sexto) de las escuelas seleccionadas se consideraron estratos, mientras que los grupos de cada grado (por ejemplo: $1 \mathrm{~A}, 1 \mathrm{~B}, 1 \mathrm{C} \ldots$ ) se consideraron conglomerados. De cada estrato, se seleccionó aleatoriamente, al menos, un grupo.

\section{Etapa 3}

Del grupo o los grupos seleccionados, se obtuvo un listado nominal de los alumnos. A cada nombre se le asignó un número progresivo e irrepetible para el grupo en cuestión, entonces, se generaron los números aleatorios necesarios para realizar el muestreo. El tamaño de muestra de cada grupo escolar seleccionado se decidió mediante asignación proporcional.

\section{Procedimiento}

De manera programada, se acudió a cada una de las escuelas seleccionadas en el muestreo. Una vez elegidos los alumnos, se citó a sus respectivos padres. En una segunda visita, se invitó a los padres a participar en el estudio; en caso de aceptar, de manera colectiva, se aplicó un cuestionario estructurado que permitía determinar la prevalencia de las enfermedades alérgicas, historia familiar de enfermedades atópicas, así como los hábitos de alimentación en los primeros meses de vida.

\section{Definiciones}

La prevalencia del asma, rinitis alérgica y dermatitis atópica se obtuvo de la respuesta afirmativa a las preguntas centrales propuestas por el International Study of Asthma and Allergies in Childhood (ISAAC): ¿Alguna vez le han diagnosticado asma a tu hijo?, ¿Alguna vez le han diagnosticado rinitis alérgica a tu hijo? y ¿Alguna vez le han diagnosticado dermatitis atópica o neurodermatitis o eczema a tu hijo?

Para los fines de este estudio, la lactancia materna prolongada fue aquella con duración mayor a 6 meses, independientemente de si era exclusiva o no; la alimentación complementaria tardía fue el momento de introducción de cualquier comida sólida o líquida, después del $4^{\circ}$ mes de vida.

\section{Análisis estadístico}

En el análisis univariante, las proporciones se compararon con la prueba de $\mathrm{X}^{2}$ (la prueba exacta de Fisher o la corrección de Yates se utilizan en caso necesario). Para comparar medias, utilizamos la prueba $t$ de Student para muestras independientes y las medianas mediante la prueba U de Mann Whitney. La asociación entre enfermedades alérgicas con lactancia materna y alimentación complementaria tardía se evaluó mediante odds ratio (OR). Se calcularon los intervalos de confianza del $95 \%$ para proporciones y OR. Mediante análisis multivariante, los factores de riesgo fueron ajustados por posibles factores de confusión (edad y sexo) con regresión logística. En el modelo, la variable dependiente fue el diagnóstico previo de la enfermedad alérgica (sí/ no). Se realizó uno para asma, rinitis alérgica y dermatitis atópica, por separado. Las covariadas en todos los modelos fueron lactancia materna prolongada, alimentación complementaria tardía, la combinación de estas dos y el antecedente de madre alérgica. La significancia estadística fue indicada por un valor de $\mathrm{p} \leq 0,05$. Los datos se analizaron en el programa IBM SPSS, versión 20.0 (IBM Co., Armonk, NY, EE.UU.) y, en Statcalc de Epi-Info para Windows.

\section{Ética}

Este estudio fue revisado y aprobado por la Comisión de Investigación y Ética de la Región Sanitaria N ${ }^{\mathrm{O}}$ VI, Secretaría de Salud, Ciudad Guzmán, Jalisco, México. Los padres de los niños firmaron, además, un consentimiento informado.

\section{RESULTADOS}

La muestra incluyó 740 niños, 393 (49,1\%) de sexo femenino. La frecuencia de madres que iniciaron la lactancia materna fue de $94,2 \%$ y el tiempo promedio que la mantuvieron fue 10,4 \pm 8,3 meses; la prevalencia de lactancia $\geq 6$ meses fue de 73,4\% y $\geq 2$ años en 11,9\% de los niños. La edad promedio de inicio de la alimentación complementaria fue a los 5,7 $\pm 2,6$ meses, a los 4 meses o antes en 31,9\% de los casos.

No se observó diferencia significativa en la frecuencia de madres que iniciaron la lactancia materna, el tiempo promedio que la mantuvieron, 
así como la frecuencia de alimentación prolongada según el tipo de enfermedad alérgica (Tabla 1). El momento de inicio de la alimentación complementaria estuvo significativamente asociado a la dermatitis atópica, sobre todo cuando esta se realizó con $\leq 4$ meses de vida. Para la frecuencia de dermatitis atópica, se notó un efecto protector de la lactancia cuando esta fue prolongada y se acompañó por una alimentación complementaria tardía ( p 0,025). Lo anterior no se documentó para asma ni rinitis alérgica.

En la Tabla 2, mediante el análisis multivariado de regresión logística, se muestra que la historia de enfermedad alérgica en la madre estuvo asociada significativamente con cualquiera de las enfermedades alérgicas en los niños, pero, solo en el caso de la dermatitis atópica, la alimentación complementaria tardía mostró ser un factor protector de su desarrollo.

\section{DISCUSIÓN}

La prevalencia de asma, rinitis alérgica y dermatitis atópica fue $8,1 \%,{ }^{15} 5,5 \%{ }^{14}$ y $3,0 \%,{ }^{16}$ respectivamente, y su comportamiento ya ha sido previamente analizado. En Ciudad Guzmán, la frecuencia de lactancia materna es inferior con respecto a la media nacional, ${ }^{17}$ y lo mismo sucede en amplias regiones de México. Entonces, de existir una asociación de riesgo entre la prevalencia de las enfermedades alérgicas y la lactancia materna, se esperaría que las primeras se incrementaran; sin embargo, tal parece que esto no ha sucedido, pues datos provenientes del Instituto Mexicano del Seguro Social, una de las instituciones de salud más importante en nuestro país, mostraron que el número de consultas otorgadas por los médicos en medicina familiar, el número de visitas al Servicio de Urgencias y el número de altas de hospital asociados con

TABLA 1. Antecedente de lactancia materna e introducción complementaria de alimentos en niños con enfermedades alérgicas*

\begin{tabular}{|c|c|c|c|c|c|c|c|c|c|c|c|c|}
\hline \multirow[b]{2}{*}{ Variable } & \multicolumn{4}{|c|}{ Asma } & \multicolumn{4}{|c|}{ Rinitis alérgica } & \multicolumn{4}{|c|}{ Dermatitis atópica } \\
\hline & $\begin{array}{c}\text { Sí } \\
(n=60)\end{array}$ & $\begin{array}{c}\text { No } \\
(n=680)\end{array}$ & $\begin{array}{c}\text { OR } \\
\text { (IC del 95\%) }\end{array}$ & $\mathrm{p}$ & $\begin{array}{c}\text { Sí } \\
(n=41)\end{array}$ & $\begin{array}{c}\text { No } \\
(n=699)\end{array}$ & $\begin{array}{c}\text { OR } \\
\text { (IC del 95\%) }\end{array}$ & $\mathrm{p}$ & $\begin{array}{c}\text { Sí } \\
(n=22)\end{array}$ & $\begin{array}{c}\text { No } \\
(n=718)\end{array}$ & $\begin{array}{c}\text { OR } \\
\text { (IC del 95\%) }\end{array}$ & $p$ \\
\hline $\begin{array}{l}\text { Edad (años), } \\
\text { media } \pm \text { DE }\end{array}$ & $9,6 \pm 1,8$ & $9,4 \pm 1,8$ & --- & 0,41 & $9,1 \pm 1,8$ & $9,5 \pm 1,8$ & --- & 0,25 & $8,9 \pm 2,1$ & $9,5 \pm 1,8$ & --- & 0,16 \\
\hline $\begin{array}{l}\text { Femenino, } \\
\mathrm{n}(\%)\end{array}$ & $\begin{array}{c}36 \\
(60,0)\end{array}$ & $\begin{array}{c}357 \\
(52,5)\end{array}$ & $\begin{array}{c}1,4 \\
(0,8-2,4)\end{array}$ & 0,26 & $\begin{array}{c}24 \\
(58,5)\end{array}$ & $\begin{array}{c}369 \\
(52,8)\end{array}$ & $\begin{array}{c}1,3 \\
(0,70-2,4)\end{array}$ & 0,47 & $\begin{array}{c}11 \\
(50,0)\end{array}$ & $\begin{array}{c}382 \\
(53,2)\end{array}$ & $\begin{array}{c}0,9 \\
(0,4-2,1)\end{array}$ & 0,77 \\
\hline $\begin{array}{l}\text { Lactancia } \\
\text { materna (sí), n (\%) }\end{array}$ & $\begin{array}{c}55 \\
(93,2)\end{array}$ & $\begin{array}{c}638 \\
(94,2)\end{array}$ & $\begin{array}{c}0,84 \\
(0,3-2,4)\end{array}$ & 0,77 & $\begin{array}{c}37 \\
(90,2)\end{array}$ & $\begin{array}{c}656 \\
(94,4)\end{array}$ & $\begin{array}{c}0,6 \\
(0,2-1,6)\end{array}$ & 0,29 & $\begin{array}{c}21 \\
(96,0)\end{array}$ & $\begin{array}{c}672 \\
(94,0)\end{array}$ & $\begin{array}{c}1,3 \\
(0,2-10,0)\end{array}$ & 0,99 \\
\hline $\begin{array}{l}\text { Lactancia } \\
\text { materna (meses), } \\
\text { mediana } \\
\text { (límite inferior - } \\
\text { límite superior) }\end{array}$ & $\begin{array}{c}8 \\
(0-36)\end{array}$ & $\begin{array}{c}8 \\
(0-48)\end{array}$ & --- & 0,31 & $\begin{array}{c}6 \\
(0-36)\end{array}$ & $\begin{array}{c}8 \\
(0-48)\end{array}$ & --- & 0,48 & $\begin{array}{c}7 \\
(0-36)\end{array}$ & $\begin{array}{c}8 \\
(0-48)\end{array}$ & --- & 0,11 \\
\hline $\begin{array}{l}\text { Edad de } \\
\text { alimentación } \\
\text { complementaria } \\
\text { (meses), mediana } \\
\text { (límite inferior - } \\
\text { límite superior) }\end{array}$ & $\begin{array}{c}6 \\
(1-12)\end{array}$ & $\begin{array}{c}6 \\
(1-24)\end{array}$ & ---- & 0,53 & $\begin{array}{c}6 \\
(2-15)\end{array}$ & $\begin{array}{c}6 \\
(1-24)\end{array}$ & ---- & 0,15 & $\begin{array}{c}4 \\
(1-6)\end{array}$ & $\begin{array}{c}6 \\
(1-24)\end{array}$ & ---- & 0,001 \\
\hline $\begin{array}{l}\text { Alimentación } \\
\text { complementaria } \\
\leq 4 \text { meses, n }(\%)\end{array}$ & $\begin{array}{c}22 \\
(37,3)\end{array}$ & $\begin{array}{c}213 \\
(31,5)\end{array}$ & $\begin{array}{c}1,3 \\
(0,7-2,3)\end{array}$ & 0,36 & $\begin{array}{c}11 \\
(26,8)\end{array}$ & $\begin{array}{c}224 \\
(32,2)\end{array}$ & $\begin{array}{c}0,8 \\
(0,4-1,6)\end{array}$ & 0,47 & $\begin{array}{c}12 \\
(54,5)\end{array}$ & $\begin{array}{c}223 \\
(31,2)\end{array}$ & $\begin{array}{c}2,6 \\
(1,1-6,7)\end{array}$ & 0,02 \\
\hline $\begin{array}{l}\text { Lactancia materna } \\
\text { prolongada y } \\
\text { alimentación } \\
\text { complementaria } \\
\text { tardía, }{ }^{+} \mathrm{n}(\%)\end{array}$ & $\begin{array}{c}24 \\
(40,7)\end{array}$ & $\begin{array}{c}349 \\
(51,6)\end{array}$ & $\begin{array}{c}0,6 \\
(0,4-1,1)\end{array}$ & 0,11 & $\begin{array}{c}23 \\
(56,1)\end{array}$ & $\begin{array}{c}350 \\
(50,4)\end{array}$ & $\begin{array}{c}1,3 \\
(0,6-2,5)\end{array}$ & 0,48 & $\begin{array}{c}6 \\
(27,3)\end{array}$ & $\begin{array}{c}367 \\
(51,5)\end{array}$ & $\begin{array}{c}0,35 \\
(0,1-0,9)\end{array}$ & 0,025 \\
\hline
\end{tabular}

* Enfermedad alérgica: asma, dermatitis atópica, rinitis alérgica.

Proporciones comparadas con prueba de $\mathrm{X}^{2}$ o exacta de Fisher o con corrección de Yates en caso necesario. Medias comparadas con prueba t de Student. Medianas comparadas mediante la prueba U de Mann Whitney.

${ }^{+}$Lactancia materna prolongada: $\geq 6$ meses. Alimentación complementaria tardía: > 4 meses. 
el asma no sufrieron incrementos sustanciales durante un período de 10 años. ${ }^{18}$ Otro estudio, que también evaluó la tendencia del asma, la rinitis alérgica y la dermatitis atópica en niños mexicanos, encontró hallazgos similares. ${ }^{19}$ Desde una perspectiva epidemiológica, pareciera que, en México, no existe asociación entre la práctica de la lactancia materna y la prevención de las enfermedades alérgicas, y nuestros resultados son consistentes con este comportamiento.

El estudio ISAAC ha evaluado, a nivel mundial, la prevalencia de las enfermedades alérgicas y los factores asociados con su desarrollo y no identificó la lactancia materna como un factor protector de la presencia actual de sibilancias, rinoconjuntivitis, dermatitis atópica o síntomas de asma grave. ${ }^{20}$ En contraste, un estudio de casocontrol realizado con niños de 3 a 7 años de edad, en el que el diagnóstico de asma, rinitis alérgica y dermatitis atópica fue confirmado clínicamente, informó que la lactancia materna exclusiva por tres meses o más era un factor protector de las enfermedades atópicas. ${ }^{21}$

Mediante nuestro estudio, no se pudo documentar el efecto protector de la lactancia materna sobre las enfermedades alérgicas, pero esto puede ser explicado por el papel que juega la herencia en su desarrollo. Una investigación con niños africanos informó que, en ausencia de predisposición genética, pero con historia de lactancia materna de manera prolongada ( $\geq 6$ meses), se constataba un efecto protector en el desarrollo de las enfermedades alérgicas. ${ }^{22}$ Igualmente, un estudio de cohorte mostró que, a la edad de 44 años, era la historia de enfermedad alérgica en la madre y no la lactancia materna exclusiva la que favorecía la posibilidad de desarrollar asma, rinitis alérgica, alergia a alimentos o sensibilización alérgica. ${ }^{23}$ Una revisión multidisciplinaria que tuvo como objetivo evaluar el impacto de la alimentación temprana en el desarrollo de las enfermedades alérgicas concluyó que la lactancia materna parecía ofrecer un efecto protector para la enfermedad atópica y que este resultado parecía ser más enérgico en los niños con herencia atópica. ${ }^{24}$ Nuestros hallazgos avalan la participación de la herencia en el origen de las enfermedades alérgicas, pues en los tres modelos analizados, la historia materna de padecimientos alérgicos permaneció como un factor asociado con

TABLA 2. Asociación de la lactancia materna prolongada más alimentación complementaria tardía con las enfermedades alérgicas

\begin{tabular}{|c|c|c|c|c|c|c|}
\hline & \multicolumn{3}{|c|}{ Modelo no ajustado } & \multicolumn{3}{|c|}{ Modelo ajustado* } \\
\hline & OR & IC del 95\% & p & OR & IC del 95\% & $\mathrm{p}$ \\
\hline \multicolumn{7}{|l|}{ Variable dependiente: asma } \\
\hline Edad & 1,1 & $0,9-1,2$ & 0,40 & --- & --- & 0,43 \\
\hline Sexo femenino & 1,5 & $0,8-2,5$ & 0,17 & --- & --- & 0,17 \\
\hline Lactancia prolongada & 0,9 & $0,3-2,1$ & 0,71 & --- & --- & 0,35 \\
\hline Alimentación complementaria tardía & 1,0 & $0,4-2,4$ & 0,94 & --- & --- & 0,49 \\
\hline $\begin{array}{l}\text { Lactancia prolongada + alimentación } \\
\text { complementaria tardía }\end{array}$ & 0,7 & $0,2-2,1$ & 0,53 & --- & --- & 0,20 \\
\hline Madre alérgica & 2,2 & $1,1-4,1$ & 0,02 & 2,1 & $1,1-4,0$ & 0,017 \\
\hline \multicolumn{7}{|l|}{ Variable dependiente: rinitis alérgica } \\
\hline Edad & 0,9 & $0,1-1,1$ & 0,35 & --- & --- & 0,32 \\
\hline Sexo femenino & 1,5 & $0,7-3,0$ & 0,24 & --- & --- & 0,22 \\
\hline Lactancia prolongada & 0,6 & $0,2-2,3$ & 0,51 & --- & --- & 0,88 \\
\hline Alimentación complementaria tardía & 0,9 & $0,2-3,0$ & 0,81 & --- & --- & 0,60 \\
\hline $\begin{array}{l}\text { Lactancia prolongada + alimentación } \\
\text { complementaria tardía }\end{array}$ & 1,7 & $0,4-7,8$ & 0,52 & --- & --- & 0,60 \\
\hline Madre alérgica & 2,9 & $1,4-6,1$ & 0,004 & 2,9 & $1,4-5,9$ & 0,004 \\
\hline \multicolumn{7}{|l|}{ Variable dependiente: dermatitis atópica } \\
\hline Edad & 0,8 & $0,7-1,1$ & 0,21 & --- & --- & 0,20 \\
\hline Sexo femenino & 0,9 & $0,4-2,3$ & 0,97 & --- & --- & 0,97 \\
\hline Lactancia prolongada & 0,9 & $0,3-3,3$ & 0,94 & --- & --- & 0,49 \\
\hline Alimentación complementaria tardía & 0,5 & $0,1-2,1$ & 0,37 & 0,36 & $0,1-0,8$ & 0,019 \\
\hline Lactancia prolongada + alimentación & & & & & & \\
\hline complementaria tardía & 0,59 & $0,1-3,4$ & 0,56 & --- & --- & 0,39 \\
\hline Madre alérgica & 2,7 & $1,1-6,9$ & 0,04 & 2,8 & $1,1-7,1$ & 0,03 \\
\hline
\end{tabular}

OR: odds ratio obtenido mediante regresión logística.

* No se muestra el OR de las variables que sirvieron de ajuste. 
su desarrollo. Consideramos que este es uno de los motivos primordiales por el cual la lactancia pareciera no ser útil para prevenirlas.

Una explicación adicional a esta falta de protección de la lactancia puede ser encontrada en el momento del inicio y tipo de alimentación complementaria. En el caso específico de la dermatitis atópica, observamos que la introducción de alimentos más allá de los 4 meses de edad e independientemente de la lactancia materna permaneció como un factor protector. Esto hace suponer que la colaboración del sistema inmune intestinal es relevante en la regulación de los mecanismos de tolerancia a alérgenos, inicialmente de origen alimenticio, y así se logrará una modificación en el desarrollo de las enfermedades alérgicas. Pues se ha visto que los niños que ingieren pescado, al menos, dos veces por semana y durante el primer año de vida tienen menor riesgo de padecer algún tipo de enfermedad alérgica y de sensibilización alérgica a los 4 años de edad. ${ }^{25}$ Lo mismo sucede con alimentos como trigo, arroz, avena, cebada o huevo, pues cuando estos son ingeridos un poco antes del tiempo correspondiente a la introducción de cada alimento, parece disminuir el riesgo de asma, rinitis alérgica y sensibilización atópica. ${ }^{26}$

Más recientemente, se mostró, en niños con dermatitis atópica, que la introducción temprana de cacahuate a la dieta disminuyó la frecuencia de alergia a este. ${ }^{27}$ Así, se abre un abanico de posibilidades para modular la respuesta inmune mediante la introducción de alimentos a la dieta. Nuestra investigación no permitió analizar a fondo las características del proceso de introducción de los diferentes alimentos a la dieta de los niños, pues solo nos limitamos a interrogar sobre la edad en la que esta fue iniciada. En consecuencia, recomendamos cautela a la hora de interpretar nuestros resultados.

Además, el tipo de alimentación durante la etapa de la lactancia y los microorganismos asociados con su uso también contribuyen al desarrollo de las enfermedades alérgicas. Un estudio de cohorte mostró que hasta una tercera parte de los niños que recibieron lactancia materna total o parcialmente tuvieron Lactobacillus en la saliva; en contraparte, ninguno de los lactantes alimentados con fórmula expresó este tipo de microorganismos. ${ }^{28}$

De igual manera sucedió con los microorganismos de la nasofaringe; los lactantes alimentados exclusivamente con leche materna tuvieron mayor presencia de Dolosigranulum y Corynebacterium, y menor cantidad de Staphylococcus y bacterias anaerobias, como Prevotella y Veillonella, comparativamente con los niños alimentados exclusivamente con fórmula. ${ }^{29}$ Cuando se evaluó la microbiota intestinal mediante la detección de microorganismos fecales, un estudio realizado en diferentes países europeos documentó que los niños con lactancia materna tenían un predominio de bifidobacterias, en tanto los que fueron alimentados con fórmula tuvieron una proporción elevada de Bacteroides y Clostridium..$^{30}$ Estas diferencias en la microbiota intestinal, producidas por el tipo de alimentación, hacen suponer la existencia de mecanismos moduladores de la respuesta inmune que pudieran intervenir en la prevención de las enfermedades alérgicas.

\section{Limitaciones}

Dentro de las limitaciones de nuestro estudio, encontramos la imposibilidad de verificar el diagnóstico de las enfermedades alérgicas. En su lugar, lo establecimos utilizando las preguntas centrales de los cuestionarios del grupo ISAAC, los que han mostrado una gran consistencia en el estudio epidemiológico de ellas. ${ }^{20}$ Otro limitante fue la dificultad de precisar la proporción de madres que utilizaron la lactancia materna como única fuente de alimentación de sus hijos, circunstancia deseable que nos permitiría analizar su papel en la prevalencia de las enfermedades alérgicas. Tampoco se midieron posibles variables confusoras, como exposición a humo de cigarro y contaminación ambiental, entre otras.

De carácter metodológico, tenemos el posible sesgo de memoria, producto del diseño retrospectivo de nuestro estudio, pues es más común que los padres de los niños con enfermedades alérgicas recuerden con mayor detalle los aspectos relacionados con la alimentación de sus hijos. Además, no se contó con un tamaño de la muestra calculado ex profeso para estimar la diferencia entre las enfermedades alérgicas en los hijos de madres alérgicas con diferentes esquemas de alimentación. A la luz de las anteriores limitaciones, nuestros resultados deben ser considerados prudentemente.

En cambio, dentro de las fortalezas metodológicas de nuestro trabajo, podemos destacar el tamaño de muestra adecuado para estimar la prevalencia de enfermedades alérgicas y la rigurosidad del muestreo, el cual fue probabilístico, estratificado y por conglomerados. Se tomó como marco muestral el total de la población de escolares de Ciudad Guzmán. 


\section{CONCLUSIONES}

Aunque la lactancia materna es reconocida por sus propiedades nutricias, por su importancia en la protección contra enfermedades infecciosas y por favorecer los vínculos afectivos entre la díada madre-hijo, no se demostró que se relacionara con la prevalencia de las enfermedades alérgicas en niños en edad escolar. En cambio, el inicio de la alimentación complementaria más allá del cuarto mes de vida sí se asoció con una disminución en la prevalencia de la dermatitis atópica.

\section{REFERENCIAS}

1. Cooper PJ, Rodrígues LC, Cruz AA, Barreto ML. Asthma in Latin America: a public heath challenge and research opportunity. Allergy 2009;64(1):5-17.

2. Aït-Khaled N, Pearce N, Anderson HR, Ellwood P, et al. Global map of the prevalence symptoms of rhinoconjunctivitis in children: The International Study of Asthma and Allergies in Childhood (ISAAC) Phase Three. Allergy 2009;64(1):123-48.

3. Arnedo-Pena A, García-Marcos L, García-Hernández G, Aguinagua-OntosoI, etal. Tendencia temporal y variaciones geográficas de la prevalencia de síntomas de rinitis alérgica en escolares de 6-7 años de ocho áreas españolas, según el ISAAC. An Pediatr (Barc) 2005;62(3):229-36.

4. GuptaR, Sheikh A,Strachan DP, Anderson HR. Timetrends in allergic disorders in the UK. Thorax 2007;62(1):91-6.

5. GruleeCG,Sanford HN. Theinfluence of breastand artificial feeding on infantile eczema. J Pediatr 1936;9(2):223-5.

6. Kull I,Wickman M, Lilja G, NordvallSL, etal. Breastfeeding and allergic diseases in infants - a prospective birth cohort study. Arch Dis Child 2002;87(6):478-81.

7. Takemura Y, Sakurai Y, Honjo S, Kusakari S, et al. Relation between breastfeeding and the prevalence of asthma: the Tokorozawa Childhood Asthma and Pollinosis Study. Am J Epidemiol 2001;154(2):115-9.

8, Savilahti E, Tainio VM, Salmenperä L, Siimes MA, et al. Prolonged exclusive breast feeding and heredity as determinants in infantile atopy. Arch Dis Child 1987;62(3):269-73.

9. Matheson MC, Erbas B, Balasuriya A, Jenkins MA, et al. Breast-feeding and atopic disease: a cohort study from childhood to middle age. J Allergy Clin Immunol 2007;120(5):1051-7.

10. Zutavern A, Von Mutius E, Harris J, Mills P, et al. The introduction of solids in relation to asthma and eczema. Arch Dis Child 2004;89(4):303-8.

11. Zutavern A, Brockow I, Schaaf B, Von Berg A, et al. Timing of solid food introduction in relation to eczema, asthma, allergic rhinitis, and food and inhalant sensitization at the age of 6 years: results from the prospective birth cohort study LISA. Pediatrics 2008;121(1):e44-52.

12. Nwaru BI, Erkkola M, Ahonen S, Kaila M, et al. Age at the introduction of solid foods during the first year and allergic sensitization at age 5 years. Pediatrics 2010;125(1):50-9.

13. Tarini BA, Carroll AE, Sox CM, Christakis DA. Systematic review of the relationship between early introduction of solid foods to infants and the development of allergic disease. Arch Pediatr Adolesc Med 2006;160(5):502-7.

14. Bedolla-Barajas M, Cuevas-Ríos G, García-Barboza E, Barrera-Zepeda AT, et al. Prevalencia y factores asociados a la rinitis alérgica en escolares de ciudad Guzmán, México. Rev Invest Clin 2010;62(3):244-51.

15. Bedolla-Barajas M, Barrera-Zepeda AT, López-Zaldo JB, Morales-RomeroJ. Asthma in Mexican school-age children is not associated with passive smoking or obesity. Asia Pac Allergy 2013;3(1):42-9.

16. Bedolla-Barajas M, Barrera-Zepeda AT, Morales-Romero J. Dermatitis atópica en niños escolares de Ciudad Guzmán, México. Prevalencia y factores asociados. Rev Alerg Mex 2010;57(3):71-8.

17. Gutiérrez JP, Rivera-Dommarco J, Shamah-Levy T, Villalpando-HernándezS, etal. Encuesta Nacional deSalud y Nutrición 2012. Resultados Nacionales. Cuernavaca: Instituto Nacional de Salud Pública;2012. [Acceso: 9 de marzo de 2015]. Disponible en: http:/ / ensanut.insp.mx/ informes/ENSANUT2012ResultadosNacionales.pdf.

18. Vargas MH,Díaz-Mejía GS, Furuya ME,Salas J, et al. Trends of asthma in Mexico: an 11-year analysis in a nationwide institution. Chest 2004;125(6):1993-7.

19. Barraza-Villarreal A,Hernández-Cadena L,Moreno-Macías $\mathrm{H}$, Ramírez-Aguilar M, et al. Trends in the prevalence of asthma and other allergic diseases in schoolchildren from Cuernavaca, Mexico. Allergy Asthma Proc 2007;28(3):368-74.

20. Björkstén B, Aït-Khaled N, Innes Asher M, Clayton TO, et al. Global analysis of breast feeding and risk of symptoms of asthma, rhinoconjunctivitis and eczema in 6-7 year old children: ISAAC Phase Three. Allergol Immunopathol (Madr) 2011;39(6):318-25.

21. Acevedo VillafañeC, Latorre LatorreF, Cifuentes Cifuentes L, Díaz-Martínez LA, etal. Influencia de la lactancia materna y la alimentación en el desarrollo de alergias en los niños. Aten Primaria 2009;41(12):675-80.

22. Obihara CC, Marais BJ, Gie RP, PotterP, etal. The association of prolonged breastfeeding and allergic disease in poor urban children. Eur Respir J 2005;25(6):970-7.

23. Matheson MC, Erbas B, Balasuriya A, Jenkins MA, et al. Breast-feeding and atopic disease: a cohort study from childhood to middle age. J Allergy Clin Immunol 2007;120(5):1051-7.

24. Van Odijk J, Kull I, Borres MP, Brandtzaeg P, et al. Breastfeeding and allergic disease: a multidisciplinary review of the literature (1966-2001) on the mode of early feeding in infancy and its impact on later atopic manifestations. Allergy 2003;58(9):833-43.

25. Kull I, Bergström A, Lilja G, Pershagen G, et al. Fish consumption during the first year of life and development of allergic diseases during childhood. Allergy 2006;61(8):1009-15.

26. Nwaru BI, Takkinen HM, Niemelä O, Kaila M, et al. Timing of infant feeding in relation to childhood asthma and allergic diseases. J Allergy Clin Immunol 2013;131(1):78-86.

27. Du Toit G, Roberts G, Sayre PH, Bahnson HT, et al. Randomized trial of peanut consumption in infants at risk for peanut allergy. N Engl J Med 2015;372(9):803-13.

28. Holgerson PL, Vestman NR, Claesson R, Ohman C, etal.Oral microbial profile discriminates breast-fed from formulafed infants. J Pediatr Gastroenterol Nutr 2013;56(2):127-36.

29. Biesbroek G, Bosch AA, Wang X, Keijser BJ, et al. Theimpact of breastfeeding on nasopharyngeal microbial communities in infants. Am J Respir Crit Care Med 2014;190(3):298-308.

30. Fallani M, Young D, Scott J, Norin E, et al. Intestinal microbiota of 6-week-old infants across Europe: geographic influence beyond delivery mode, breastfeeding, and antibiotics. J Pediatr Gastroenterol Nutr 2010;51(1):77-84. 